\title{
Tbx 1 regulates Vegfr3 and is required for lymphatic vessel development
}

\author{
Li Chen, ${ }^{1,4}$ Annalisa Mupo, ${ }^{5}$ Tuong Huynh, ${ }^{4}$ Sara Cioffi, ${ }^{5}$ Matthew Woods, ${ }^{2}$ Chengliu Jin, ${ }^{3}$ Wallace McKeehan, ${ }^{3}$ \\ LuAnn Thompson-Snipes, ${ }^{2}$ Antonio Baldini, ${ }^{4,6}$ and Elizabeth Illingworth ${ }^{4,7,8}$ \\ 'Program of Cardiovascular Sciences and 2Department of Pathology, Baylor College of Medicine, Houston, TX 77030 \\ ${ }^{3}$ Mouse Genetics Core Laboratory and ${ }^{4}$ Institute of Biosciences and Technology, Center for Cancer and Stem Cell Biology, Texas A\&M University Health Science Center, \\ Houston, TX 77030 \\ ${ }^{5}$ European School of Molecular Medicine, 80131 Naples, Italy \\ Institute of Genetics and Biophysics, Consiglio Nazionale delle Ricerche, 80131 Naples, Italy \\ 7 Universita degli Studi di Salerno, 84084 Fisciano, Italy \\ ${ }^{8}$ Dulbecco Telethon Institute, Telethon Institute of Genetics and Medicine, 80131 Naples, Italy
}

$$
\text { L }
$$

ymphatic dysfunction causes several human diseases, and tumor lymphangiogenesis is implicated in cancer spreading. TBX1 is the major gene for DiGeorge syndrome, which is associated with multiple congenital anomalies. Mutation of $T b x 1$ in mice recapitulates the human disease phenotype. In this study, we use molecular, cellular, and genetic approaches to show, unexpectedly, that $T b x 1$ plays a critical role in lymphatic vessel development and regulates the expression of Vegfr3, a gene that is essential for lymphangiogenesis. Tbx 1 activates Vegfr3 transcription in endothelial cells (ECs) by binding to an enhancer element in the Vegfr3 gene. Conditional deletion of Tbx 1 in ECs causes widespread lymphangiogenesis defects in mouse embryos and perinatal death. Using the mesentery as a model tissue, we show that $T b x 1$ is not required for lymphatic EC differentiation; rather, it is required for the growth and maintenance of lymphatic vessels. Our findings reveal a novel pathway for the development of the lymphatic vessel network.

\section{Introduction}

The lymphatic vascular system is involved in many pathological processes, including lymphedema and the metastatic spread of cancer. Despite the importance of the lymphatic system for human health, our knowledge of lymphatic vessel development lags considerably behind that of blood vascular development, and it is only in recent years that some of the molecules that regulate lymphangiogenesis have been identified (for review see Tammela et al., 2005). The data presented in this study establish the transcription factor TBX1 as a new player in lymphangiogenesis.

$T B X 1$ encodes a $T$ box transcription factor involved in the 22q11 deletion syndrome (22q11DS), also known as DiGeorge syndrome. $22 q 11 D S$ patients have a complex phenotype that

L. Chen and A. Mupo contributed equally to this paper.

Correspondence to Elizabeth Illingworth: eillingworth@dti.telethon.it

Abbreviations used in this paper: 22q11DS, 22q11 deletion syndrome; $\beta$-gal, $\beta$-galactosidase; ChIP, chromatin immunoprecipitation; EC, endothelial cell; $G I_{\text {, }}$ gastrointestinal; HMLEC, human microvascular LEC; HUVEC, human umbilical vein EC; LEC, lymphatic EC; qRT-PCR, quantitative real-time PCR; TBE, T boxbinding element; $T M$, tamoxifen. includes cardiovascular defects, craniofacial dysmorphism, hypocalcemia, immune deficiency, behavioral disorders, and psychiatric diseases. Most of the physical abnormalities are caused by TBX1 haploinsufficiency (Yagi et al., 2003), and we have shown that TBX1 may contribute to the psychiatric disorders (Paylor et al., 2006). Lymphatic defects have not been reported in 22q11DS patients, with a single exception (Mansir et al., 1999). However, the recurrent infections associated with the disease may be contributed by subtle lymphatic abnormalities in addition to the known $\mathrm{T}$ cell immune deficiency.

In the mouse, mutation of Tbxl recapitulates the common physical abnormalities seen in patients (Jerome and Papaioannou, 2001; Lindsay et al., 2001; Merscher et al., 2001). During mouse development, $T b x 1$ is widely expressed in the developing pharynx, ear, and the endothelial lining of some blood vessels (Vitelli et al., 2002; Paylor et al., 2006). Intriguingly, a

(C) 2010 Chen et al. This article is distributed under the terms of an AttributionNoncommercial-Share Alike-No Mirror Sites license for the first six months after the publication date (see http://www.rupress.org/terms). After six months it is available under a Creative Commons License (Attribution-Noncommercial-Share Alike 3.0 Unported license, as described at http://creativecommons.org/licenses/by-nc-sa/3.0/). 
variety of vascular abnormalities has been reported in 22q11DS patients (D'Antonio and Marsh, 1987; MacKenzie-Stepner et al., 1987; Mansour et al., 1987). In this study, we sought to establish the requirement for $T b x 1$ in endothelial cells (ECs) and found an unexpected and critical role in lymphatic vessel development.

\section{Results and discussion}

\section{Loss of Tbx 1 in ECs causes perinatal}

\section{lethality and Iymphatic abnormalities}

We deleted Tbxl specifically in ECs using an EC-specific Cre driver, Tie2-Cre (Kisanuki et al., 2001). We crossed Tie2-Cre; Tbx $1^{\text {lacz/+ }}$ mice with Tbx $1^{\text {flox/flox }}$ mice and genotyped 114 offspring at weaning. We did not recover any Tie2-Cre; Tbx flox/lacz pups (i.e., null for Tbxl in ECs and heterozygous in all other tissues). In contrast, this genotype was recovered at the normal Mendelian ratio (10/39) on embryonic day (E) 18.5. We established that Tie2-Cre; Tbx $1^{\text {flox/lacz }}$ mutants died between postnatal day (P) 2 and 4, when they showed growth failure, dehydration, and abdominal chylous ascites (Fig. $1 \mathrm{~A}^{\prime}$ ), which are features that were not present in controls (Fig. $1 \mathrm{~A}$ ).
A similar pathology has been reported in several mouse mutants that have mutations in genes involved in lymphatic vessel development (for review see Tammela et al., 2005), suggesting that $T b x 1$ may be required in ECs for lymphangiogenesis. In view of the dramatic phenotype seen in Tie2-Cre; Tbx $1^{\text {flox/lacz }}$ mutants, we first evaluated lymphangiogenesis in the gastrointestinal (GI) tract of preterm embryos. For this, we performed immunostaining on isolated GI tracts of E18.5 embryos using antibodies against three EC-specific genes, CD31 (Pecam-1), Vegfr3, and Lyve1. Anti-Pecam-1 identifies ECs of arteries, veins, and lymphatic vessels, whereas anti-Vegfr3 and antiLyve1 are specific for lymphatic ECs (LECs) at this embryonic stage. Anti-Pecam-1 revealed an overall reduction in the number of vessels in the mesentery of both Tie2-Cre; Tbx $1^{\text {flox/lacz }}$ mutants (Fig. 1, B and B') and Tbxl laczlacz (null) mutants (not depicted). We used anti-Vegfr3 to confirm that the missing vessels were indeed lymphatic vessels (Fig. 1, C and $\mathrm{C}^{\prime}$ ). Similar results were obtained with anti-Lyve1 (Fig. S1), thereby confirming that the lack of staining with anti-Vegfr3 was caused by the absence of the lymphatic vessels. Thus, EC-specific loss of Tbxl causes a developmental failure of the entire GI lymphatic vasculature.
Figure 1. Lymphatic abnormalities in ECspecific Tbx 1-null mutants. (A) In control embryos, chyle is visible in mesenteric lymphatic vessels (arrowheads). ( $\left.\mathrm{A}^{\prime}\right)$ On P4, Tie2-Cre; $T b \times l^{\text {flox/lacz }}$ mutants show growth retardation, abdominal distention, and accumulation of chyle in the intestinal wall (arrows) and membranous mesentery (arrowheads). (B and $B^{\prime}$ ) Immunohistochemistry on isolated intestines at E18.5. Anti-Pecam-1 shows that Tie2Cre; Tbx $7^{\text {flox/lacz }}$ mutants $\left(B^{\prime}\right)$ have fewer mesenteric vessels than controls (B). $\left(C^{\prime}\right)$ Anti-Vegfr3 reveals that

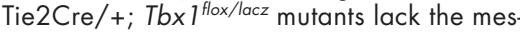
enteric lymphatic vessels. (C) Control mesentery is shown. L, lymphatic vessel; A, artery; $V$, vein; I, intestine. Bars, $1 \mathrm{~mm}$.
Tie2-Cre;Tbx $1^{\text {flox } /+}$
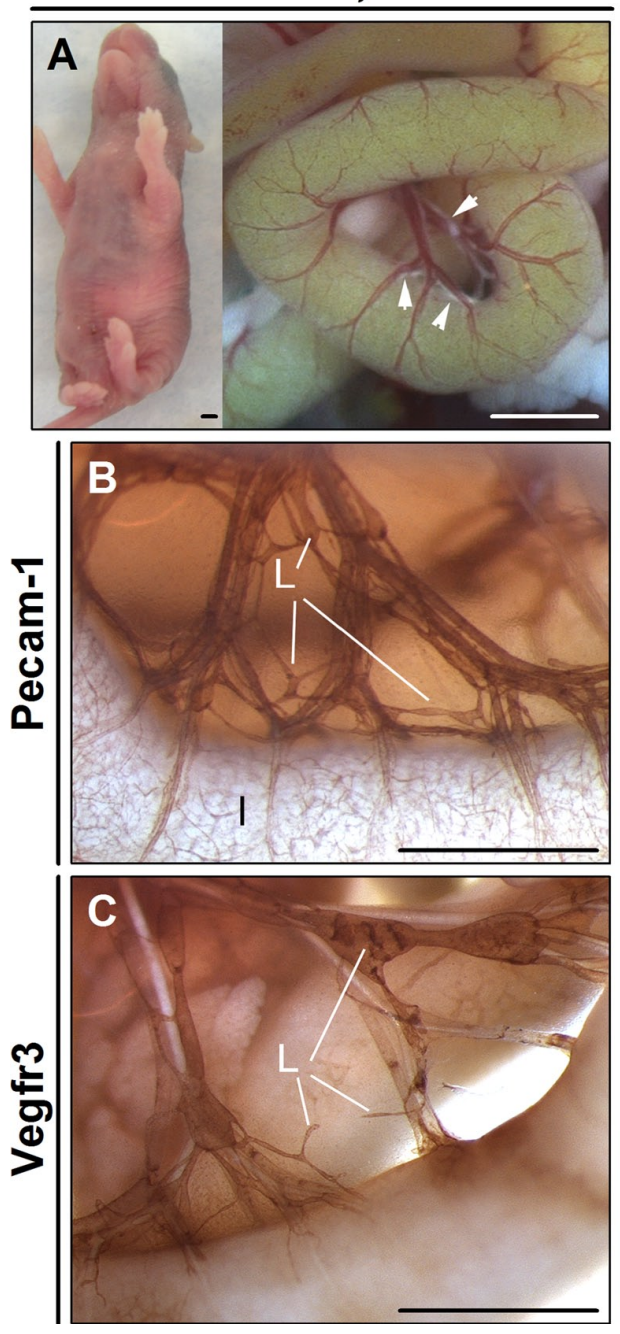

Tie2-Cre; Tbx $1^{\text {flox/acz }}$
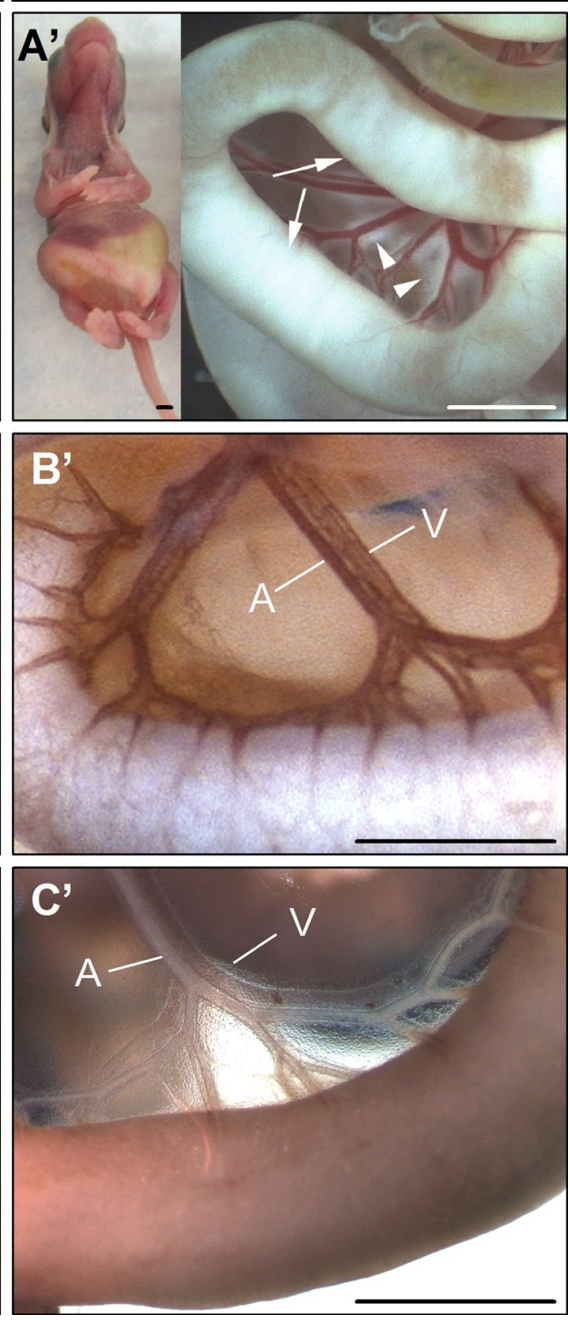
We next examined lymphatic vessels in other tissues, including heart, diaphragm, and skin in $T b x 1^{\text {lacz/+ }}$ and $T b x 1^{\text {lacz/acz }}$ embryos at E18.5 (Fig. S2). In the heart, anti-Vegfr3 immunostaining revealed a severe reduction in the number of lymphatic vessels in $T b x I^{\text {laczlacz }}$ embryos, with only a few vessels adjacent to the aorta being identifiable (Fig. S2, A-B'). Anti-Pecam-1 immunostaining showed similar staining patterns in both genotypes (Fig. S2, C and $\mathrm{C}^{\prime}$ ), although in Tbx $1^{\text {laczlacz }}$ embryos, some vessels were dilated, and there was a reduced vessel density. In the diaphragm, an extensive network of lymphatic vessels was observed in control embryos (Fig. S2, D and E), whereas in Tbx $1^{\text {laczlacz }}$ embryos, this network was severely reduced (Fig. S2, D' and $E^{\prime}$ ). Similar results were obtained with antiLyve1 (Fig. S2, F and $\mathrm{F}^{\prime}$ ). In the skin, Tbxl laczlacz embryos had fewer subcutaneous lymphatic vessels than control embryos (Fig. S2, $\mathrm{G}^{\prime}$ and $\mathrm{H}^{\prime}$ and $\mathrm{G}$ and $\mathrm{H}$, respectively). Consistent with this observation, Tbxi $1^{\text {laczllacz }}$ embryos showed subcutaneous edema at E14.5 (Fig. S2, I and I').

Tbx 1 is required early in mesenteric Iymphangiogenesis

Because of the severity of the mesenteric phenotype, we focused our attention on the function of Tbxl in this tissue. In the mouse, the first mesenteric lymphatic vessels form around E13 (van der Putte, 1975; Kim et al., 2007). To monitor Tbxl expression, we used a lacZ reporter allele (Lindsay et al., 2001) and an antiTbx1 antibody. At E13.5, we identified $\beta$-galactosidase ( $\beta$-gal) activity in the proximal mesentery, where it colocalized with anti-Vegfr3 immunostaining (Fig. 2, A and B), indicating that Tbxl is expressed in the LECs of early mesenteric lymphatic vessels. Note that $T b x 1$ expression was not LEC specific at this or later embryonic stages, as $\beta$-gal activity was also identifiable in some ECs of the mesenteric artery and vein. Robust $\beta$-gal activity in lymphatic vessels continued through E16.5 (Fig. 2, C, D, and F). Anti-Tbx1 immunostaining showed a similar pattern of expression (Fig. 2 E). We determined the time requirement for $T b x 1$ in mesenteric lymphangiogenesis using transgenic mice that carry a tamoxifen (TM)-inducible Cre recombinase under the control of a ubiquitous promoter (TgCAGG-CreER ${ }^{\mathrm{TM}}$; Hayashi and McMahon, 2002). TgCAGG$\mathrm{CreER}^{\mathrm{TM}}$; Tbx $1^{\text {lacz/+ }}$ male mice were bred with $T b x 1^{\text {flox/flox }}$ females. Pregnant females were injected with TM between E11.5 and E14.5 to effect Cre-induced excision of the floxed Tbxl exon 5, and thereby gene inactivation between E12.5 and E15.5 (see Materials and methods). Mice with the genotype TgCAGG-CreER ${ }^{\mathrm{TM}} ;$ Tbx $1^{\text {flox/lacz }}$ constituted the test group, whereas embryos with the genotype TgCAGG-CreER ${ }^{\mathrm{TM}} ; \mathrm{Tb} x \mathrm{I}^{\text {flox/+ }}$ constituted the control group. Embryos were collected at E18.5, and intestines were immunostained with anti-Vegfr3. In control embryos exposed to TM at E11.5, the mesenteric lymphatics
TbX1 lacz/+

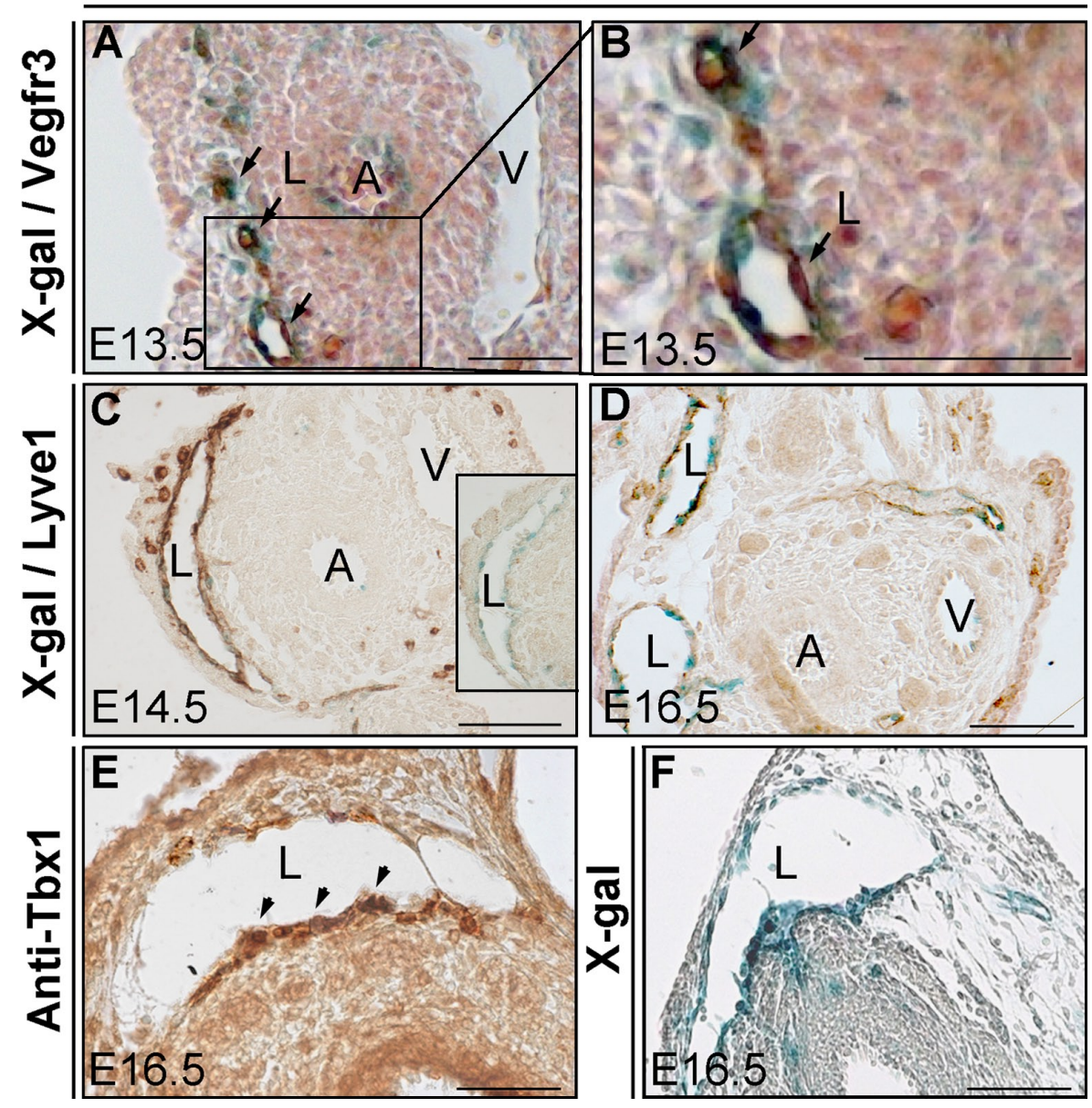

Figure 2. Tbx 1 colocalizes with LEC-specific markers in mesenteric lymphatic vessels. (A and B) LacZ reporter activity revealed by X-gal staining (blue) colocalizes with antiVegfr3 immunostaining (brown) in mesenteric lymphatic vessels (arrows) of $T b x 7^{l a c z /+} \mathrm{em}$ bryos at E13.5. The boxed area in $A$ is magnified in B. (C and D) Colocalization of LacZ reporter activity and anti-Lyve 1 immunostaining in Tbx $1^{\text {lacZ/+ }}$ embryos at E14.5 (C) and E16.5 (D). (C, inset) X-gal staining on an adjacent section is shown. (E and F) Tbxl protein is expressed in mesenteric LECs at E16.5 ( $E$, arrowheads) and has a similar distribution to the LacZ reporter activity at this stage (F). A, mesenteric artery; $V$, mesenteric vein; L, lymphatic vessel. Bars, $100 \mu \mathrm{m}$. 


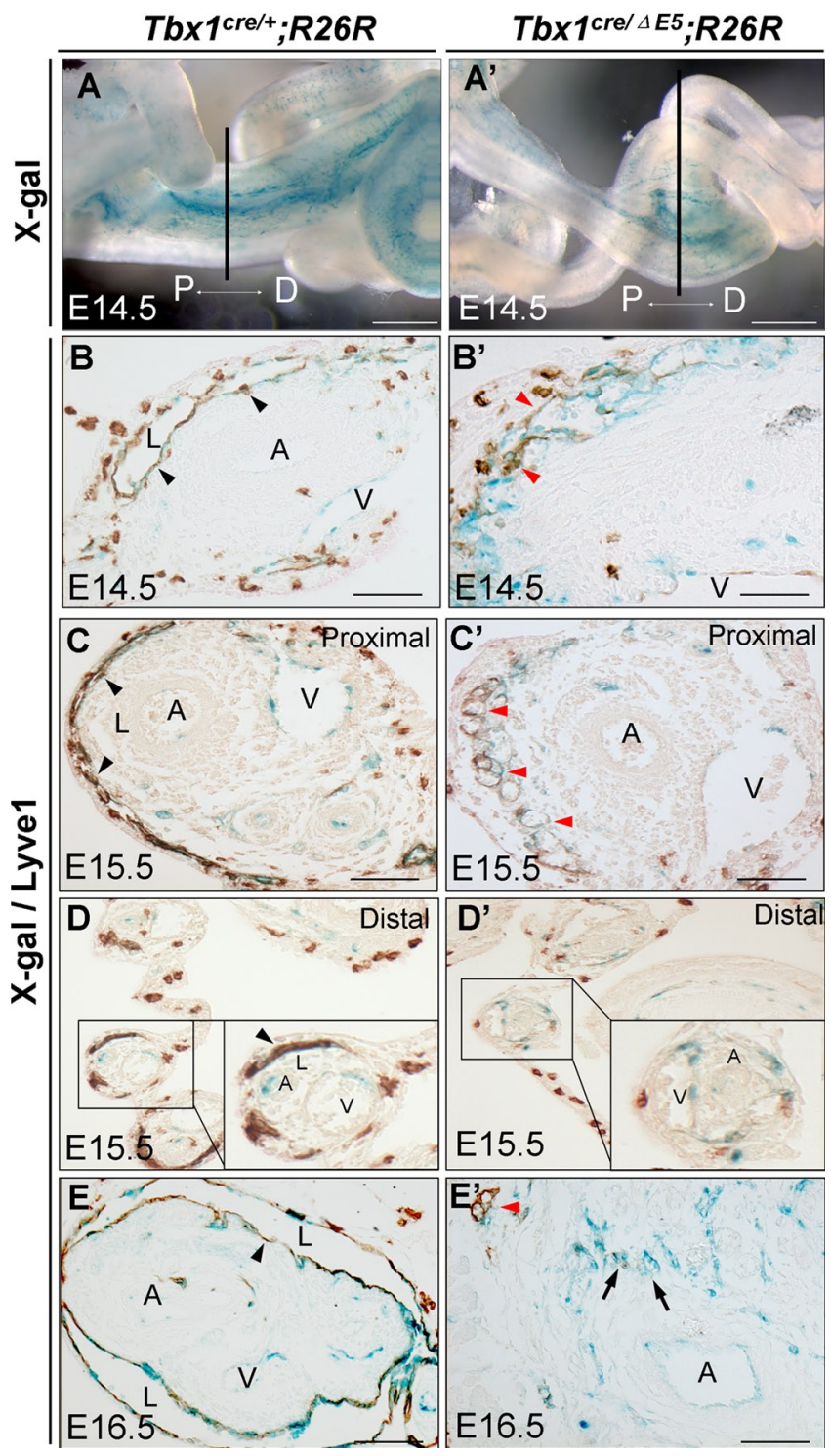

Figure 3. Fate mapping of Tbx 1 -expressing cells in the mesentery. (A and $A^{\prime}$ ) $X$-gal staining of isolated intestines at E14.5 shows extensive contribution of $T b \times 7$-traced cells to the mesenteric vessels of control $\left(\mathrm{A} ; \mathrm{Tb} \times{ }^{\mathrm{Cre} /+}\right.$; $R 26 R)$ and mutant $\left(T b x 7^{\text {Cre/ } / \triangle E 5} ; R 26 R\right)$ embryos $\left(A^{\prime}\right)$. Black lines indicate the angle of sections in $B$ and $B^{\prime}$. ( $\left(B\right.$ and $\left.B^{\prime}\right)$ In the proximal mesentery of E14.5 embryos, X-gal staining and anti-Lyvel colocalize in mesenteric LECs in control (B) and mutant ( $\left.\mathrm{B}^{\prime}\right)$ embryos. Note the abnormal anatomy of the mutant lymphatic vessels ( $B^{\prime}$, red arrowheads). (C and $\left.C^{\prime}\right) A t E 15.5$, in the proximal mesentery, highly abnormal lymphatic vessels in mutant embryos $\left(C^{\prime}\right.$, red arrowheads) are both $\mathrm{X}_{\text {-gal }}{ }^{+}$and Lyve $\mathrm{l}^{+}$. (D and $\left.\mathrm{D}^{\prime}\right)$ In the distal mesentery, lymphatic vessels were present in control embryos $\left(D\right.$, arrowhead) but not mutant embryos $\left(D^{\prime}\right)$. Insets show the boxed area at higher magnification. (E and $\left.E^{\prime}\right)$ At E 16.5, very few $X$-gal ${ }^{+} /$Lyve $^{+}{ }^{+}$vessels are identifiable $\left(E^{\prime}\right.$, red arrowhead). Note that the vasa vasorum ( $E^{\prime}$, black arrows) is $X$-gal ${ }^{+} /$Lyve 1 negative. $A$, mesenteric artery; $V$, mesenteric vein; L, lymphatic vessel; P, proximal; D, distal. Black arrowheads indicate lymphatic vessels. Bars, $100 \mu \mathrm{m}$.

developed normally (Fig. S3 A). In contrast, TgCAGG-CreER ${ }^{\mathrm{TM}}$; Tbx $1^{\text {flox/lacz }}$ mutants exposed to TM at E11.5 (Fig. S3 B) and E12.5 (not depicted) had no identifiable mesenteric lymphatics at E18.5, which is similar to Tie2-Cre; $T b x l^{\text {flox/lacz }}$ and $T b x l^{\text {lacz/lacz }}$ mutants. Induction at E13.5 resulted in near-complete absence of lymphatic vessels, although in two out of seven TgCAGG$\mathrm{CreER}^{\mathrm{TM}} ; \mathrm{Tb} x 1^{\text {flox/lacz }}$ mutants analyzed, a few Vegfr $3^{+}$vessels were seen (Fig. S3 C, arrows). Induction at E14.5 resulted in apparently normal lymphatic vessel development at E18.5 (Fig. S3 D). Thus, Tbxl is required for lymphangiogenesis until E14. In the mouse, this is $\sim 24 \mathrm{~h}$ after the onset of mesenteric lymphangiogenesis (van der Putte, 1975). As shown in Fig. 2 (A and B), Tbxl is expressed in mesenteric LECs at this critical stage.

\section{Tbx 1 is required for lymphatic vessel}

\section{maintenance}

We asked whether Tbxl-deficient lymphatic vessels form, and if so, what their fate is. To address this, we used two previously characterized $T b x l$-null alleles, $T b x l^{\Delta E S}$ (Xu et al., 2004) and Tbx $I^{\text {Cre }}$ (Huynh et al., 2007), in conjunction with the Cre reporter R26R (Soriano, 1999). X-gal staining of isolated intestines at E14.5 shows extensive contribution of Tbxl-traced cells to the mesenteric vessels of control ( $\mathrm{Tbxl}^{\mathrm{Cre} /+}$; R26R) embryos (Fig. $3 \mathrm{~A}$ ) and null mutant (TbxI ${ }^{\text {Cre/ } \triangle E 5}$; R26R) embryos (Fig. $3 \mathrm{~A}^{\prime}$ ). Double staining (X-gal and anti-Lyve1) of $\mathrm{Tbxl}^{\mathrm{Cre} / \mathrm{+}}$; R26R embryos at E14.5 (Fig. 3 B) and E15.5 (Fig. 3 C) revealed that the mesenteric lymphatic endothelium is largely composed of $T b x 1$-traced cells (Fig. 3, black arrowheads). In $T b x I^{\text {Cre/AES}}$; R26R embryos (Fig. 3, B' and $\mathrm{C}^{\prime}$ ), Tbxl-traced cells contributed to clusters of vessels (Fig. 3, B' and $\mathrm{C}^{\prime}$, red arrowheads) that were unlike the characteristic network of lymphatic vessels seen in controls. Nevertheless, some of these abnormal vessels were Lyve $1^{+}$, indicating their lymphatic nature. In the distal mesentery, $\beta$-gal ${ }^{+}$ vessels were Lyve1 negative (Fig. 3, D and $\mathrm{D}^{\prime}$ ) and are likely tributaries of the mesenteric artery and vein (note that the punctate brown signals in mutant and control sections are $\mathrm{Lyve}^{+}$macrophages). Thus, $T b x l$ is not essential for mesenteric lymphatic vessel formation. We determined the fate of the abnormal lymphatic vessels at later embryonic stages. At E16.5, only a few $\beta$-gal ${ }^{+}$vessels were identifiable in the proximal mesentery of $T b x I^{\text {Crel } \triangle E S}$; R26R embryos (Fig. $3 \mathrm{E}^{\prime}$ ), some of which were also Lyve $^{+}$(Fig. $3 \mathrm{E}^{\prime}$, red arrowhead), whereas in the distal mesentery, $\beta$-gal ${ }^{+}$vessels were Lyve1 negative, as seen at E15.5 (not depicted). At E18.5, $\beta-\mathrm{gal}^{+}$vessels were Lyve1 negative and had the anatomy of veins and arteries (unpublished data). Similar results were obtained at the aforementioned embryonic stages with anti-Vegfr3 (unpublished data). Thus, on a Tbxl-null background, the mesenteric lymphatic vessels form, but they are abnormal, they fail to extend into the distal mesentery, and between E15.5 and E16.5, they are almost completely lost. To determine whether this loss is caused by cell death, we performed anti-caspase 3 immunostaining on isolated GI tracts of $T b x l^{l a c Z /+}$ and Tbx $1^{\text {lacZlacZ }}$ embryos at E14.5 and E16.5. Results showed a significant increase in the number of apoptotic cells adjacent to the mesenteric artery of $T b x I^{\text {lacZlacZ }}$ embryos $(\mathrm{P}<0.01)$ at E16.5 (Fig. S3, F-G) but not at E14.5 (Fig. S3, E, E', and G).

\section{Tbx 1 regulates transcription of growth} factors and angiogenic markers in ECs We next used quantitative real-time PCR (qRT-PCR) to explore the transcriptional response of 82 genes involved in angiogenesis and lymphangiogenesis (RT ${ }^{2}$ Profiler PCR Array; QIAGEN) to $T B X 1$ expression in cultured human umbilical vein ECs 
A
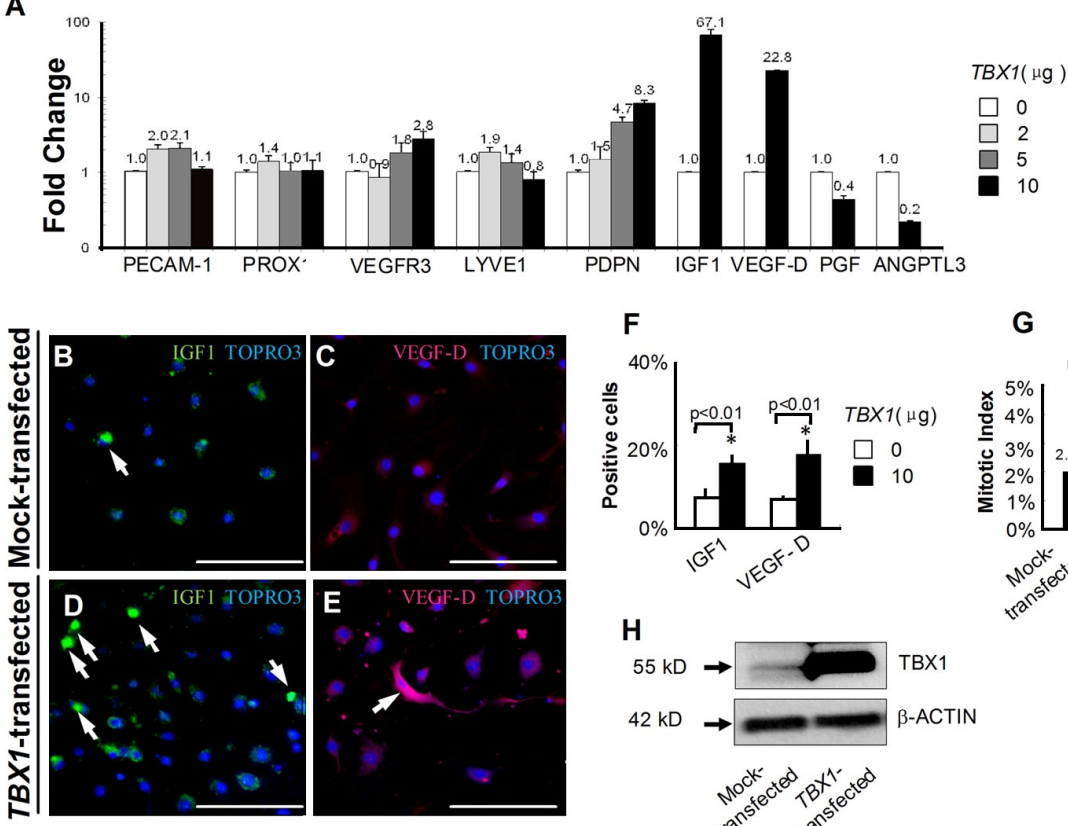

G

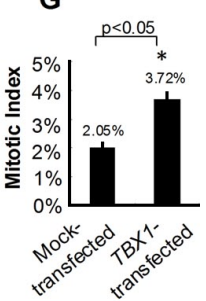

H

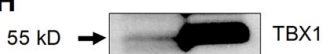

$42 \mathrm{kD}$

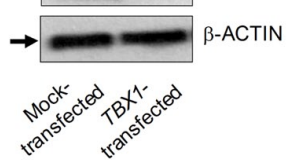

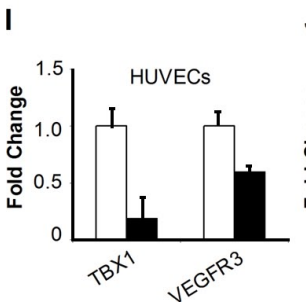

J
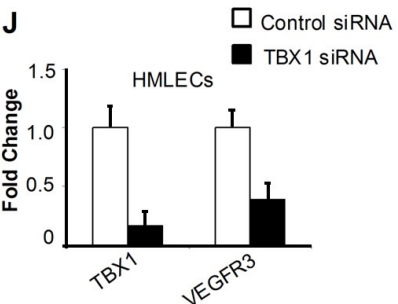

K

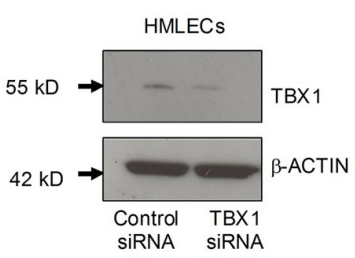

Figure 4. Tbxl activates prolymphangiogenic genes in ECs. (A) Fold change histogram representation of qRT-PCR results of RNA from HUVECs transfected with increasing amounts of TBXI expression vector. (B-E) Anti-IGF 1 (green) and anti-VEGFD (red) staining of TBX 1transfected HUVECs (D and E) and control cultures $(B$ and $C)$. Arrows indicate $\mid G F]^{+}$and VEGFD ${ }^{+}$cells. Mock transfections (B, C, and F [white columns]) were performed by transfecting HUVECs with empty vector. TOPRO3 identifies cell nuclei. (F) More IGF $1^{+}$and VEGFD ${ }^{+}$ cells were present in $T B X 7$-transfected cultures compared with control cultures. 500 cells were counted in three independent experiments for each antibody. (G) Cell proliferation in TBX1-transfected HUVECs increased $>1.8$ fold above that of control cultures. (H) Western blotting reveals increased TBX1 protein expression after $T B X 1$ transfection of HUVECs. (I and J) TBXI and VEGFR3 mRNA levels in TBX 1-siRNA- treated or control siRNA-treated HUVECs (I) and HMLECs (J) were quantified by qRT-PCR and normalized to GAPDH. Control expression levels were set to 1 . In both cell types, TBXI and VEGFR3 mRNA levels were greatly reduced after $T B X 1$ knockdown. Values are mean \pm SEM. $(K)$ Western blotting with antibodies to TBXI and $\beta$-actin showed that TBXI protein expression was reduced in HMLECs treated with TBX1-siRNA. Bars, $100 \mu \mathrm{m}$.
(HUVECs). Among the markers of lymphangiogenesis, PROX1 and $L Y V E 1$ did not change significantly in response to $T B X 1$ transfection. However, VEGFR3, PDPN, and VEGFD increased significantly, as did IGF1 (Fig. 4 A). We validated data obtained by qRT-PCR and immunocytofluorescence with VEGFD and IGF1 antibodies on TBX1-transfected HUVECs (Fig. 4, B-E). Results showed that $\sim 7 \%$ of the control cell population expressed VEGFD or IGF1 protein, and this percent increased two- to threefold after TBX1 transfection (Fig. 4 F). This suggests that at least part of the increased level of mRNA encoding those proteins is caused by an increase in the number of cells expressing those markers. We evaluated cell proliferation by immunocytofluorescence with anti-phosphohistone H3. In HUVECs transfected with $3 \mu \mathrm{g}$ human TBXI expression vector, cell proliferation increased $>1$.8-fold above that of control cultures (Fig. $4 \mathrm{G}$ ). We used Western blotting to confirm that transfection of HUVECs with the TBX1-expressing vector resulted in increased expression of TBX1 protein (Fig. $4 \mathrm{H}$ ).

\section{Tbx 1 regulates VEGFR3 in ECs}

Of the three most highly up-regulated genes, IGF1, VEGFD, and $P D P N$, only $P d p n$ loss of function in mice is associated with lymphatic defects, namely lymphatic vessel dilatation and impaired lymphatic transport (Schacht et al., 2003), which is unlike the lymphatic phenotype of $T b x 1$-null mutants. In addition, anti-VEGFD immunostaining in Tbxl-null mutants was normal. Specifically, it was expressed in LECs lining the jugular lymph sacs and in subcutaneous lymphatic vessels (unpublished data), both of which are present in $T b x 1^{-/-}$mutants, although they are abnormal and reduced in number.

Therefore, we focused our attention on VEGFR3, which is essential for lymphangiogenesis (Karkkainen et al., 2000, 2001) and is regulated by TBX1 in a dose-dependent manner (Fig. 4 A). Because HUVECs express endogenous TBX1, we knocked it down using siRNA to test whether $V E G F R 3$ expression responds to it. We obtained an $\sim 80 \%$ reduction in TBXI expression and a $40 \%$ reduction in VEGFR3 expression in multiple experiments (Fig. 4 I). Similar experiments performed on cultured human microvascular LECs (HMLECs) yielded a $60 \%$ reduction in VEGFR3 expression (Fig. $4 \mathrm{~J}$ ). siRNA knockdown of TBX1 mRNA resulted in reduced TBX1 protein expression in both HMLECs (Fig. $4 \mathrm{~K}$ ) and in HUVECs (not depicted).

To identify putative TBX1-responsive enhancers within the VEGFR3 gene, we searched for evolutionarily conserved DNA segments containing conserved $\mathrm{T}$ box-binding elements (TBE). A 1.1-kb segment was identified that encompassed intron 11-12 and contained a single TBE. We cloned this DNA fragment from the mouse genome and named it $\operatorname{In}^{11-12 T B E}$. We asked whether the $\operatorname{In}^{11-12 \mathrm{TBE}}$ enhancer responds to Tbxl in a tissue culture system. To test this, we cotransfected JEG3 cells with a $T b x 1$ expression vector and a luciferase reporter containing $\mathrm{In}^{11-12 \mathrm{TBE}}$ and a basal promoter (Fig. 5 A). Overexpression of $T b x l$ led to a fourfold increase in luciferase activity in multiple experiments (Fig. 5 B). In contrast, mutation of TBE 
Figure 5. Tbx 1 regulates VEGFR3 expression via a conserved TBE in the endogenous VEGFR3 gene. (A) Schematic of the In 11-12TBE luciferase reporter. A mutant version of the reporter construct was generated by site-directed mutagenesis of the TBE located within the putative VEGFR3 enhancer $\left(\operatorname{In}^{11-12 T B E}\right)$. (B) Cotransfection of JEG3 cells with the luciferase reporter and increasing amounts of $T b x l$ expression vector increased activity of the luciferase reporter containing the wild-type enhancer (wt-TBE) but not the mutant enhancer (mut-TBE). Control cultures were transfected with empty ex pression vector. (C) PCR of chromatin immunoprecipitated from HUVECs. Antibodies used for immunoprecipitation are indicated above the panel. A chromatin sample immunoprecipitated with a nonspecific antibody (c-Myc) served as a negative control and the input sample as a positive control. Samples immunoprecipitated with anti-Tbxl showed enrichment of the VEGFR3 enhancer containing the conserved TBE. (D-E') Histological sections of the mesentery of transgenic embryos carrying the wild-type (D and $E$ ) or mutant TBE ( $D^{\prime}$ and $E^{\prime}$ ) enhancer construct at E15.5. Colocalization of $\beta$-gal expression and anti-Vegfr3 immunostaining was seen in mesenteric LECs of transgenic embryos carrying the wild-type TBE (D and $E$, arrowheads) but not with the mutated TBE ( $D^{\prime}$ and $\left.E^{\prime}\right)$. Arrows indicate the mesenteric artery (A). $V$, mesenteric vein; L, lymphatic vessel. Bars, $100 \mu \mathrm{m}$.
A

In ${ }^{11-12 T B E}$ luciferase reporter

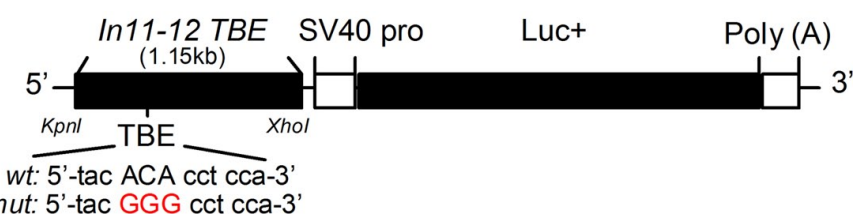

mut: 5'-tac GGG cct cca-3'

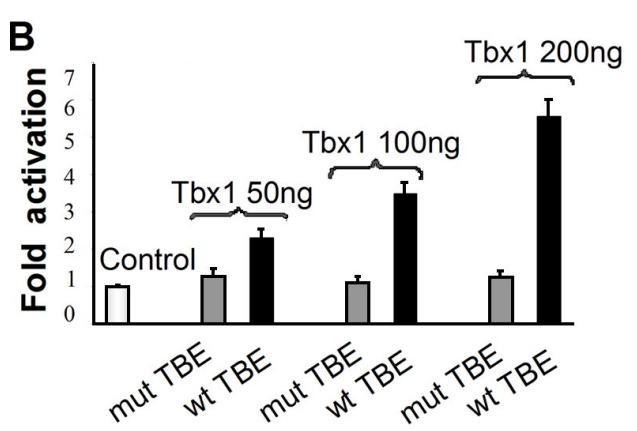

C

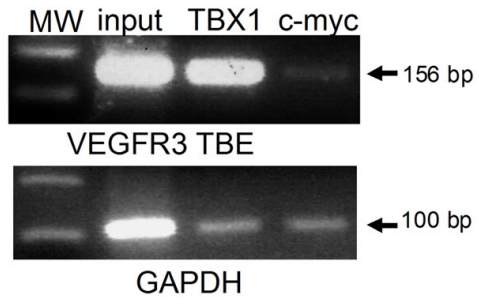

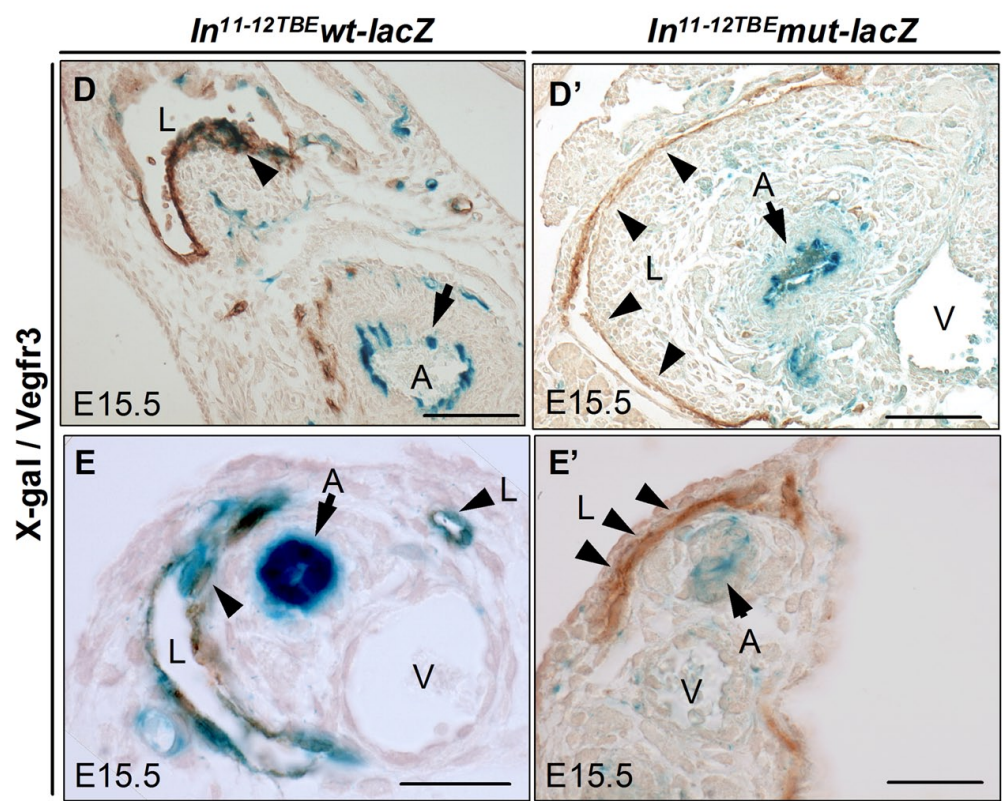

(see Materials and methods) made this enhancer unresponsive to Tbx1 (Fig. 5 B), demonstrating that its activation is mediated by this TBE.

We next determined whether this TBE is occupied by endogenous TBX1 using chromatin immunoprecipitation (ChIP) from HUVECs, which express endogenous $T B X 1$ and VEGFR3. DNA fragments precipitated by anti-TBX1 were purified and tested for the presence of the aforementioned TBE by PCR. Results showed enrichment of DNA fragments containing the TBE in the ChIP sample (Fig. 5 C), indicating that TBX1 occupies this site in these cells.

Next, to test whether $\operatorname{In}^{11-12 \mathrm{TBE}}$ functions as an LEC enhancer in vivo, we generated transient transgenic mice carrying an HSP-lacZ reporter driven by $\operatorname{In}^{11-12 T B E}$ with either the wildtype or mutated TBE (see Materials and methods). We obtained 10 transgenic embryos with the wild-type transgene $\left(\mathrm{In}^{11-12 \mathrm{TBE}} \mathrm{wt}-\right.$ lacZ) at E15.5, of which five were $\beta$-gal ${ }^{+}$. In these embryos, $\beta$-gal-expressing cells colocalized with anti-Vegfr3 in LECs of the mesenteric lymphatic vessels (Fig. 5, D and E) and in lymphatic vessels of the skin and heart (not depicted). We also generated transgenic embryos carrying $\operatorname{In}^{11-12 \mathrm{TBE}}$ with the TBE mutated. We obtained 11 transgenic embryos with the mutant transgene ( In $^{11-12 T B E}$ mut-lacZ) at E15.5, of which five were $\beta$-gal ${ }^{+}$. In these embryos, no $\beta$-gal-expressing cells were seen in Vegfr $3^{+}$mesenteric LECs (Fig. 5, $\mathrm{D}^{\prime}-\mathrm{E}^{\prime}$ ) or in skin or heart lymphatic vessels (not depicted), indicating that the mutated transgene was not activated in these cells. These results demonstrate that $\mathrm{In}^{11-12 \mathrm{TBE}}$ functions as an LEC enhancer in vivo and that its activity depends on its TBE. In transgenic embryos carrying the wild-type or mutated TBE, we observed strong X-gal staining in ECs of the mesenteric artery (Fig. 5, D-E'). Vegfr3 is not normally expressed in arterial ECs at this stage, suggesting that the endogenous Vegfr3 gene harbors a suppressor element that is absent in the transgene.

In summary, our data show that $T b x 1$ is required in ECs for development of the GI lymphatic vasculature. In mice, the loss of this population of lymphatic vessels is lethal after birth. Elsewhere, lymphatic vessels develop in both Tie2-Cre; $T b x l^{f f o x} / a c z$ mutants and in $T b x l$ germline-null mutants, although in the tissues surveyed, they were much reduced in number and abnormal. 
Thus, Tbxl is not essential for lymphangiogenesis per se; rather, it is required for the development of the lymphatic network. We have gained insights into when and how Tbxl functions in mesenteric lymphatic development. On a Tbxl-null background, lymphatic vessels form at the appropriate time, but they are highly disorganized and fail to extend distally. Between E15.5 and E16.5, these abnormal lymphatic vessels are lost, most likely as a result of cell death. This suggests that Tbxl has at least two roles in the mesentery. First, it is required for growth and expansion of the lymphatic network. This is consistent with our data from cultured LECs that show increased cell proliferation in response to Tbxl transfection. Second, it is required to maintain mesenteric lymphatic vessels.

In a search for potential effectors of these functions, we found that the expression of Vegfr3 is not maintained in $T b x I^{\text {laczlacz }}$ mutants or in Tie2-Cre; Tbx $1^{\text {flox/lacz }}$ mutants. Through tissue culture and in vivo genetic experiments, we have demonstrated that Vegfr 3 is a direct transcriptional target of Tbx 1 and identified a Tbx1-responsive element in the Vegfr3 gene. Thus, we have directly linked Tbx1 to an essential lymphangiogenesis signaling pathway.

Our data support the hypothesis that, in the absence of $T b x 1$, lymphatic vessels are not maintained, secondary to a failure to maintain Vegfr3 expression LECs. This hypothesis is consistent with in vitro and in vivo studies that have shown a critical role for VEGF-C/Vegfr3 signaling in the growth, migration, and survival of LECs (Mäkinen et al., 2001a,b; Veikkola et al., 2001).

Could loss of Vegfr3 in Tbxl-null ECs account for the entire lymphatic phenotype of Tbxl-null mutants? In the future, it will be interesting to see whether forced expression of Vegfr3 in ECs rescues lymphatic defects in Tbxl mutants.

How is Tbxl regulated? There is very little information to date. A Forkhead (Fox) transcription factor-responsive element has been identified upstream of the $T b x l$ gene (Yamagishi et al., 2003). The enhancer responds in tissue culture to Foxc2, which has also been involved in lymphatic development (for review see Tammela et al., 2005). However, it is unknown whether Foxc2 is required for Tbxl expression in endothelium, and, in any case, the phenotypes resulting from Tbxl and Foxcl or Foxc2 loss of function are different. In particular, Foxc1/2 has a later role in lymphangiogenesis compared with Tbxl (Norrmén et al., 2009).

TBX1 mutations can cause DiGeorge syndrome, but lymphatic defects have not been reported as part of the clinical spectrum associated with this disease. As the human disease is caused by heterozygous mutations, it is possible that a single copy of $T B X 1$ is sufficient to support normal lymphatic vessels. Alternatively, the defects may be subclinical. Our findings should stimulate the search for subtle defects in patients. Possible consequences of mild lymphatic abnormalities in the GI tract may be reduced absorption of lipids and resulting growth delay, a common finding in affected infants (Ryan et al., 1997; Scambler, 2000; Emanuel et al., 2001). Another question raised by this study is whether $T B X 1$ plays a role in cancer. Many studies have linked tumor lymphangiogenesis to the development of lymph node metastases, and VEGFR3 inhibitors are currently being developed as anticancer agents. It would be interesting to investigate whether VEGFR3 expression in the vicinity of solid tumors is regulated by $T B X 1$.

\section{Materials and methods}

\section{Mouse lines}

The following mouse lines were used: Tbx $1^{\text {lacz/t+ }}$ (Lindsay et al., 2001), Tbx $7^{f l o x /+}$ (Xu et al., 2004), Tie2Cre (Kisanuki et al., 2001), R26R (Soriano, 1999), TgCAGG-CreER ${ }^{\text {TM }}$ (Hayashi and McMahon, 2002), Tbx $7^{\Delta E 5 /+}$ (Xu et al., 2004), and Tbx $7^{\mathrm{Cre} /+}$ (Huynh et al., 2007). Genotyping was performed according to the aforementioned original reports. Activation of CAGG-CreER ${ }^{\text {TM }}$ was performed with a single intraperitoneal injection of $75 \mathrm{mg} / \mathrm{kg}$ body weight TM (Xu et al., 2005).

$X$-gal staining, immunostaining, and immunocytofluorescence

$\beta$-Gal activity was revealed on 4\% PFA-fixed samples by X-gal staining. Samples were photographed directly or paraffin embedded and sectioned at $10 \mu \mathrm{m}$. Immunostaining and immunocytofluorescence were performed using the following antibodies: rat anti-Vegfr3 (eBioscience), rat antiPECAM-1 (BD), rabbit anti-Lyvel (Abcam), monoclonal anti-VEGFD (R\&D Systems), and rabbit anti-IGF 1 (Santa Cruz Biotechnology, Inc.). Nonfluorescent antibodies were visualized after mounting slides in Cytoseal (Thermo Fisher Scientific). Fluorescent antibodies were visualized using imaging medium (Vectashield; Vector Laboratories). Whole-mount specimens were photographed using a dissecting microscope (Stemi 2000; Carl Zeiss, Inc.) equipped with a camera (Axiocam; Carl Zeiss, Inc.) and the manufacturer's acquisition software. Images of histological sections were acquired using a microscope (Axioskop 40; Carl Zeiss, Inc.) equipped with a camera (Axiocam; Carl Zeiss, Inc.) and the manufacturer's acquisition software. Photoshop (Adobe) was used for trimming and labeling images.

\section{Cell manipulations}

HUVECs (Lonza) were electroporated with increasing amounts of a TBX1 expression or with empty vector and an EGFP reporter to monitor transfection. Transfection of a standard amount of DNA in all samples was achieved by adjusting the quantity of empty vector in the mix. After $24 \mathrm{~h}$, cells were fixed in 4\% PFA for immunocytofluorescence or lysed for RNA isolation. qRT-PCR was performed using commercial primers (RT2Profiler PCR Array; PAHS-024; human angiogenesis; QIAGEN).

RNA interference was performed using commercial siRNA for TBXl (ON-TARGETplus SMARTpool; Thermo Fisher Scientific) and a control siRNA (Thermo Fisher Scientific). HUVECs or HMLECs were transfected with $40 \mathrm{nM}$ siRNA and Lipofectamine 2000 (Invitrogen). TBX1 mRNA was evaluated by GRT-PCR, and TBXI protein was evaluated by Western blotting with anti-Tbxl (Invitrogen). Protein levels were normalized to anti$\beta$-actin (Sigma-Aldrich).

\section{Enhancer activity assays}

For the luciferase construct, a 1.1-kb of DNA surrounding a conserved TBE in the Vegfr3 intron 11-12 was PCR amplified with primers 5'-CGAGACTGGAAGGAGGTGACC-3' and 5'-CACCAGCTTGCTCACCGTC-3'. The amplicon, named $\operatorname{In}^{11-12 T B E}$, was first cloned into a T-vector, pCRII-TOPO (Invitrogen), and after Kpnl-Xhol digestion, it was subcloned into a pGL3 luciferase expression vector (Promega). Subsequently, the enhancer fragment was subjected to site-directed mutagenesis of the TBE. The native sequence 5'-TACACACCTCCA-3' was mutated to 5'-TACGGGCCTCCA-3' using a site-directed mutagenesis kit (QuikChange XL; Agilent Technologies). A $T b x l$ expression vector was cotransfected with the enhancer reporter (wild type or mutated) into JEG3 cells, which were harvested $48 \mathrm{~h}$ later. JEG3 cells transfected with empty expression vector, and without the enhancer reporter, constituted the control sample. Experiments were performed in triplicate and repeated twice.

In 11-12TBE $w$ t-HSPlacZ and In $^{11-12 T B E}$ mut-HSPlacZ transgenes were injected into $\mathrm{FVB} / \mathrm{NCr}$ pronuclei. Embryos were harvested at E15.5, stained with $X$-gal, and genotyped by PCR with the following primers: TT_LaczF, 5'-CTGGAATTCCGCCGATACT-3'; TT_LaczR, 5'-CCGGATTGATGGTAGTGGTC-3'; TT_VR3F, 5'-GCCTCTGTTCTGGGTAGACG-3'; and TT_VR3R, 5'-AGTGCTGCCTCTGACCTCAT-3'

\section{ChIP}

ChIP experiments were performed as described previously (Breiling et al., 2004). Chromatin from cross-linked HUVECs cells was immunoprecipitated 
with anti-Tbxl (Invitrogen) or with anti-c-Myc (control; Sigma-Aldrich). DNA purified from chromatin was quantified by qRT-PCR using the following primers: TBE_ip_F, 5'-CACACCTCCAGAGCCTCTTC-3'; TBE_ip_R, 5'-CCCTGCCTATCATCCAAAGA-3'; TBE_ip_F 1, 5'-CTGAACAAAGGCACCCACTT-3'; and TBE_ip_R 1, 5'-GGGGAGGAGAGAGGTCCATA-3'.

\section{Online supplemental material}

Fig. S1 shows Lyvel immunostaining of E18.5 GI tracts of Tbx1 mutants. Fig. S2 shows that Tbxl is expressed in and is required for development of lymphatic vessels of the heart, diaphragm, and skin. Fig. S3 shows that a lymphatic phenotype after time-controlled Tbx 1 mutagenesis increased apoptosis in the proximal mesentery of $T b \times 1$-null mutants. Online supplemental material is available at http://www.jcb.org/cgi/content/full/jcb.200912037/DCl.

We thank Dr. Yanagisawa for the Tie2-Cre line and Dr. Huansheng $X_{U}$, A. Leeming, P. Terrell, S. Tang, G. Ji, and W. Yu for valuable technical assistance.

This work was funded by the Associazione Italiana per la Ricerca sul Cancro (E. Illingworth), the National Institute of Mental Health (E. Illingworth) and the Dulbecco Telethon Institute (E. Illingworth).

Submitted: 7 December 2009

Accepted: 5 April 2010

\section{References}

Breiling, A., L.P. O'Neill, D. D'Eliseo, B.M. Turner, and V. Orlando. 2004 Epigenome changes in active and inactive polycomb-group-controlled regions. EMBO Rep. 5:976-982. doi:10.1038/sj.embor.7400260

D'Antonio, L.D., and J.L. Marsh. 1987. Abnormal carotid arteries in the velocardiofacial syndrome. Plast. Reconstr. Surg. 80:471-472.

Emanuel, B.S., D. McDonald-McGinn, S.C. Saitta, and E.H. Zackai. 2001. The 22q11.2 deletion syndrome. Adv. Pediatr. 48:39-73.

Hayashi, S., and A.P. McMahon. 2002. Efficient recombination in diverse tissues by a tamoxifen-inducible form of Cre: a tool for temporally regulated gene activation/inactivation in the mouse. Dev. Biol. 244:305-318. doi:10.1006/dbio.2002.0597

Huynh, T., L. Chen, P. Terrell, and A. Baldini. 2007. A fate map of Tbx1 expressing cells reveals heterogeneity in the second cardiac field. Genesis. 45:470-475. doi:10.1002/dvg.20317

Jerome, L.A., and V.E. Papaioannou. 2001. DiGeorge syndrome phenotype in mice mutant for the T-box gene, Tbxl. Nat. Genet. 27:286-291. doi: $10.1038 / 85845$

Karkkainen, M.J., R.E. Ferrell, E.C. Lawrence, M.A. Kimak, K.L. Levinson, M.A. McTigue, K. Alitalo, and D.N. Finegold. 2000. Missense mutations interfere with VEGFR-3 signalling in primary lymphoedema. Nat. Genet. 25:153-159. doi:10.1038/75997

Karkkainen, M.J., L. Jussila, R.E. Ferrell, D.N. Finegold, and K. Alitalo. 2001. Molecular regulation of lymphangiogenesis and targets for tissue oedema. Trends Mol. Med. 7:18-22. doi:10.1016/S1471-4914(00)01864-5

Kim, K.E., H.K. Sung, and G.Y. Koh. 2007. Lymphatic development in mouse small intestine. Dev. Dyn. 236:2020-2025. doi:10.1002/dvdy.21200

Kisanuki, Y.Y., R.E. Hammer, J. Miyazaki, S.C. Williams, J.A. Richardson, and M. Yanagisawa. 2001. Tie2-Cre transgenic mice: a new model for endothelial cell-lineage analysis in vivo. Dev. Biol. 230:230-242. doi:10.1006/ dbio. 2000.0106

Lindsay, E.A., F. Vitelli, H. Su, M. Morishima, T. Huynh, T. Pramparo, V. Jurecic, G. Ogunrinu, H.F. Sutherland, P.J. Scambler, et al. 2001. Tbx1 haploinsufficieny in the DiGeorge syndrome region causes aortic arch defects in mice. Nature. 410:97-101. doi:10.1038/35065105

MacKenzie-Stepner, K., M.A. Witzel, D.A. Stringer, W.K. Lindsay, I.R. Munro, and H. Hughes. 1987. Abnormal carotid arteries in the velocardiofacial syndrome: a report of three cases. Plast. Reconstr. Surg. 80:347-351. doi:10.1097/00006534-198709000-00002

Mäkinen, T., L. Jussila, T. Veikkola, T. Karpanen, M.I. Kettunen, K.J. Pulkkanen, R. Kauppinen, D.G. Jackson, H. Kubo, S. Nishikawa, et al. 2001a. Inhibition of lymphangiogenesis with resulting lymphedema in transgenic mice expressing soluble VEGF receptor-3. Nat. Med. 7:199-205. doi:10.1038/84651

Mäkinen, T., T. Veikkola, S. Mustjoki, T. Karpanen, B. Catimel, E.C. Nice, L. Wise, A. Mercer, H. Kowalski, D. Kerjaschki, et al. 2001b. Isolated lymphatic endothelial cells transduce growth, survival and migratory signals via the VEGF-C/D receptor VEGFR-3. EMBO J. 20:4762-4773. doi:10.1093/emboj/20.17.4762

Mansir, T., D. Lacombe, T. Lamireau, L. Taine, J.F. Chateil, B. Le Bail, J.L. Demarquez, and M. Fayon. 1999. Abdominal lymphatic dysplasia and 22q11 microdeletion. Genet. Couns. 10:67-70.
Mansour, A.M., R.B. Goldberg, F.M. Wang, and R.J. Shprintzen. 1987. Ocular findings in the velo-cardio-facial syndrome. J. Pediatr. Ophthalmol. Strabismus. 24:263-266.

Merscher, S., B. Funke, J.A. Epstein, J. Heyer, A. Puech, M.M. Lu, R.J. Xavier, M.B. Demay, R.G. Russell, S. Factor, et al. 2001. TBX1 is responsible for cardiovascular defects in velo-cardio-facial/DiGeorge syndrome. Cell. 104:619-629. doi:10.1016/S0092-8674(01)00247-1

Norrmén, C., K.I. Ivanov, J. Cheng, N. Zangger, M. Delorenzi, M. Jaquet, N. Miura, P. Puolakkainen, V. Horsley, J. Hu, et al. 2009. FOXC2 controls formation and maturation of lymphatic collecting vessels through cooperation with NFATc1. J. Cell Biol. 185:439-457. doi:10.1083/ jcb.200901104

Paylor, R., B. Glaser, A. Mupo, P. Ataliotis, C. Spencer, A. Sobotka, C. Sparks, C.H. Choi, J. Oghalai, S. Curran, et al. 2006. Tbx1 haploinsufficiency is linked to behavioral disorders in mice and humans: implications for 22q11 deletion syndrome. Proc. Natl. Acad. Sci. USA. 103:7729-7734. doi:10.1073/pnas.0600206103

Ryan, A.K., J.A. Goodship, D.I. Wilson, N. Philip, A. Levy, H. Seidel, S. Schuffenhauer, H. Oechsler, B. Belohradsky, M. Prieur, et al. 1997. Spectrum of clinical features associated with interstitial chromosome 22q11 deletions: a European collaborative study. J. Med. Genet. 34:798804. doi:10.1136/jmg.34.10.798

Scambler, P.J. 2000. The 22q11 deletion syndromes. Hum. Mol. Genet. 9:24212426. doi:10.1093/hmg/9.16.2421

Schacht, V., M.I. Ramirez, Y.K. Hong, S. Hirakawa, D. Feng, N. Harvey, M. Williams, A.M. Dvorak, H.F. Dvorak, G. Oliver, and M. Detmar. 2003. T1alpha/podoplanin deficiency disrupts normal lymphatic vasculature formation and causes lymphedema. EMBO J. 22:3546-3556. doi:10.1093/emboj/cdg342

Soriano, P. 1999. Generalized lacZ expression with the ROSA26 Cre reporter strain. Nat. Genet. 21:70-71. doi:10.1038/5007

Tammela, T., T.V. Petrova, and K. Alitalo. 2005. Molecular lymphangiogenesis: new players. Trends Cell Biol. 15:434-441. doi:10.1016/j.tcb.2005 .06 .004

van der Putte, S.C. 1975. The early development of the lymphatic system in mouse embryos. Acta Morphol. Neerl. Scand. 13:245-286.

Veikkola, T., L. Jussila, T. Makinen, T. Karpanen, M. Jeltsch, T.V. Petrova, H. Kubo, G. Thurston, D.M. McDonald, M.G. Achen, et al. 2001 Signalling via vascular endothelial growth factor receptor-3 is sufficient for lymphangiogenesis in transgenic mice. EMBO J. 20:1223-1231. doi:10.1093/emboj/20.6.1223

Vitelli, F., M. Morishima, I. Taddei, E.A. Lindsay, and A. Baldini. 2002. Tbx mutation causes multiple cardiovascular defects and disrupts neural crest and cranial nerve migratory pathways. Hum. Mol. Genet. 11:915-922. doi:10.1093/hmg/11.8.915

Xu, H., M. Morishima, J.N. Wylie, R.J. Schwartz, B.G. Bruneau, E.A. Lindsay, and A. Baldini. 2004. Tbx1 has a dual role in the morphogenesis of the cardiac outflow tract. Development. 131:3217-3227. doi:10.1242/dev.01174

$\mathrm{Xu}, \mathrm{H}$., F. Cerrato, and A. Baldini. 2005. Timed mutation and cell-fate mapping reveal reiterated roles of Tbx 1 during embryogenesis, and a crucial function during segmentation of the pharyngeal system via regulation of endoderm expansion. Development. 132:4387-4395. doi:10.1242/dev.02018

Yagi, H., Y. Furutani, H. Hamada, T. Sasaki, S. Asakawa, S. Minoshima, F. Ichida, K. Joo, M. Kimura, S. Imamura, et al. 2003. Role of TBX1 in human del22q11.2 syndrome. Lancet. 362:1366-1373. doi:10.1016/ S0140-6736(03)14632-6

Yamagishi, H., J. Maeda, T. Hu, J. McAnally, S.J. Conway, T. Kume, E.N. Meyers, C. Yamagishi, and D. Srivastava. 2003. Tbx1 is regulated by tissue-specific forkhead proteins through a common Sonic hedgehogresponsive enhancer. Genes Dev. 17:269-281. doi:10.1101/gad.1048903 